\title{
Orphan symptoms in advanced cancer patients followed at home
}

\author{
Sebastiano Mercadante • Giampiero Porzio - Alessandro Valle • \\ Flavio Fusco • Federica Aielli • Claudio Adile • Alessandra Casuccio • \\ On behalf of "Home Care Italy" group (HOCAI group)
}

Received: 14 May 2013 / Accepted: 1 October 2013 /Published online: 13 October 2013

(C) Springer-Verlag Berlin Heidelberg 2013

\begin{abstract}
Orphan symptoms are rarely assessed, particularly at home. The aim of this multicenter prospective study was to assess the prevalence of these symptoms and eventual factors possibly associated in advanced cancer patients at admission of a home care program. A prospective study was performed at three home care programs in Italy. Patients' data were collected, including age, sex, diagnosis, and Karnofsky status. Possible contributing factors were analyzed; preexisting neurological diseases, cerebral metastases, hyperthermia, diabetes, a state of dehydration clinically evident and/or oliguria, possible biochemical parameters when available, data regarding recent chemotherapy, opioids and doses, use of neuroleptics, benzodiazepine or anticonvulsants, corticosteroids, anti-inflammatory, and antibiotics were collected. Myoclonus, hiccup, sweating, pruritus, and tenesmus, either rectal or vesical, were assessed, according to a preliminary definition, at time of home care program admission. Three hundred sixty-two patients were surveyed at the three home care programs. Globally, 48 patients presented one or more orphan symptoms in the period taken into consideration,
\end{abstract}

S. Mercadante $(\bowtie) \cdot$ F. Fusco $\cdot$ C. Adile

Pain Relief and Palliative Care Unit, La Maddalena Cancer Center,

Via San Lorenzo 312, 90146 Palermo, Italy

e-mail: terapiadeldolore@lamaddalenanet.it

S. Mercadante

Palliative Medicine, Department of Anesthesia, \& Intensive Care,

University of Palermo, Palermo, Italy

G. Porzio · F. Aielli

Home care program, L' Aquila per la vita, L'Aquila, Italy

A. Valle

Home care program, Fondazione FARO, Torino, Italy

A. Casuccio

Department of Sciences for Health Promotion and Mother-Child

Care "G. D'Alessandro", University of Palermo, Palermo, Italy and 7 patients presented more than 1 symptom. One patient presented occasional and diffuse myoclonus. Nineteen patients presented sweating, 13 patients presented pruritus, and 14 patients presented hiccup. Finally, nine patients presented rectal or vesical tenesmus. There was a significant correlation between sweating and transdermal fentanyl use $(P=0.044)$, fever $(P=0.001)$, hiccup $(P<0.0005)$, and vesical tenesmus $(P=0.028)$. Pruritus was not associated to any factor. Hiccup was associated with gender (males, $P=0.006$ ) and sweating $(P<0.0005)$. Vesical tenesmus was associated with fever ( $P=0.019)$ and sweating $(P=0.028)$. Although the symptoms examined have a low prevalence in advanced cancer patients admitted to home care, the distress for patients may be high and deserve further analyses. Given the low prevalence of these symptoms, large studies are needed to find possible associated factors.

Keywords Palliative care $\cdot$ Home care $\cdot$ Myoclonus · Sweating $\cdot$ Pruritus $\cdot$ Hiccup $\cdot$ Tenesmus

\section{Introduction}

Symptom assessment is of paramount importance to recognize the priority of treatment in palliative care. The most popular and validated tool for symptom assessment is the Edmonton Symptom Assessment System [3, 13, 14]. Implementation of the ESAS provided improvements in symptom screening, symptom control, and functional assessment [6]. However, there is a group of symptoms which is seldom evaluated in most symptom assessment tools [5], and can be considered as orphan symptoms. At the best of our knowledge, no epidemiological or clinical study exists regarding the prevalence of sweating, hiccup, myoclonus, pruritus, and tenesmus in patients followed by a home palliative care team. The Home Care-Italy group has been recently established 
with the intent to implement the information on cancer patients followed at home, given the paucity of existing data in this setting. The aim of this multicenter prospective study was to assess the prevalence of these symptoms and eventual factors possibly associated in advanced cancer patients at admission to a home care program.

\section{Patients and methods}

A prospective study was performed at three home care programs in Italy. A consecutive sample of patients admitted to home care programs in Turin (FARO foundation), L'Aquila (L'Aquila per la vita), and Genua (ASL 6 genovese, Genua) were surveyed for a period of 6 months. A preliminary investigation meeting by web was performed to homogenize the definitions and the way to assess these symptoms, as well as to identify simple factors possibly associated with these symptoms (see Appendix). Informed consent and institutional approval were obtained. Patients' data were collected, including age, sex, diagnosis, and Karnofsky status. In a preliminary consensus, it was decided, on the basis of reliability and repeatability in the home setting, to explore the following factors which potentially would have influence the occurrence of orphan symptoms: preexisting neurological diseases, cerebral metastases, hyperthermia, diabetes, a state of dehydration clinically evident and/or oliguria, and possible biochemical parameters when available. The choice was based on the simplicity of data to be recorded at home and available facilities. Moreover, data regarding recent chemotherapy, opioids and doses, use of neuroleptics, benzodiazepine or anticonvulsants, corticosteroids, anti-inflammatory, and antibiotics were collected.

Myoclonus, hiccup, sweating, pruritus, and tenesmus, either rectal or vesical, were assessed at time of home care program admission. Myoclonus was considered as a brief, involuntary twitching of a muscle or a group of muscles. Characteristics investigated were only nocturnal, occasionally, persistent through the day, limited to few muscular groups, or diffused $[7,8,11]$. Sweating was considered as the production of fluids secreted by the sweat glands in the skin [2]. Characteristics were nocturnal, persistent, relevant to need frequent dress changes, and clearly consequential to drug administration. Pruritus was considered as a sensation that causes the desire or reflex to scratch and was assessed as localized, diffuse, limited in time, persisting, associated with skin infection or lesions, and allergy [16, 18]. Hiccup was defined as an involuntary contraction (myoclonic jerk) of the diaphragm that may repeat several times per minute, involving a reflex arc. Once triggered, the reflex causes a strong contraction of the diaphragm followed about $0.25 \mathrm{~s}$ later by closure of the vocal cords, which results in the classic "hic" sound. Hiccups may occur individually, or they may occur in bouts. The rhythm of the hiccup, or the time between hiccups, tends to be relatively constant. Hiccup was considered as occasional, persisting, or continuous $[12,13]$. Finally, tenesmus was considered as a sensation of fullness or incomplete relief during urination or defecation $[14,15]$.

Statistical analysis

Data were collected and analyzed by the SPSS Software 14.0 version (SPSS, Inc., Chicago, IL, USA). Descriptive summaries for all measures are reported as means and standard deviations (SD) for numeric variables, and as percentages for categorical variables. Statistical analysis of quantitative data, included descriptive statistics, was performed for all the items. The chi-square test was used to make comparisons with respect to categorical variables, and the Fisher exact test was used if sample size criteria were not met for chi-square approximation. The one-way analysis of variance was used for parametric analysis. All $P$ values were two-sided and $P$ values less than 0.05 were considered to indicate statistical significance.

\section{Results}

Three hundred sixty-two patients were surveyed at the three home care programs. Primary diagnoses were in a rank order as follows: gastrointestinal 71 , lung 54 , genitourinary 54 , breast 36 , liver 28 , pancreas 22 , hematological malignancies 16, head and neck 12, and others 69 . The mean age was 73 years (SD 13.3), the mean Karnofsky at admission was 50.1 (SD 17.4), and 179 were males.

Globally, 48 patients presented one or more orphan symptoms in the period taken into consideration, and 7 patients presented more than 1 symptom.

One patient presented occasional and diffuse myoclonus. Nineteen patients presented sweating, 13 patients presented pruritus, and 14 patients presented hiccup. Finally, nine patients presented rectal or vesical tenesmus. The characteristics of these symptoms are reported in Table 1.

There was a significant correlation between sweating and transdermal fentanyl use $(P=0.044)$, fever $(P=0.001)$, hiccup $(P<0.0005)$, and vesical tenesmus $(P=0.028)$. Pruritus was not associated to any factor taken into consideration. Hiccup was associated with gender (males, $P=0.006$ ) and sweating $(P<0.0005)$. Vesical tenesmus was associated with fever $(P=0.019)$ and sweating $(P=0.028)$.

\section{Discussion}

Literature regarding orphan symptoms, like myoclonus, sweating, pruritus, hiccup, as well as tenesmus, is scarce, and this is 
Table 1 Prevalence of orphan symptoms and their characteristics

\begin{tabular}{ll}
\hline & Number (\%) \\
\hline Myoclonus & $1(0.27 \%)$ \\
Nocturnal & 1 \\
Occasionally & 1 \\
Persistent & - \\
Limited & \\
Diffuse & \\
Sweating & $19(5.24 \%)$ \\
Nocturnal & 8 \\
Persistent & 5 \\
Relevant & 9 \\
Drug-related & 3 \\
Pruritus & $13(3.59 \%)$ \\
Localized & 7 \\
Diffuse & 5 \\
Short lived & 5 \\
Persisting & 7 \\
Skin lesions & \\
Allergy or drug-related & \\
Hiccup & $14(3.86 \%)$ \\
Occasional & 8 \\
Persisting & 5 \\
Continuous & 1 \\
Tenesmus & $9(2.48 \%)$ \\
Rectal & 6 \\
Vesical & 3 \\
\hline
\end{tabular}

even more evident in patients followed at home by a palliative care team. Furthermore, assessment tools have never been assessed.

Myoclonus may develop in response to infection, head or spinal cord injury, stroke, stress, brain tumors, kidney or liver failure, lipid storage disease, chemical or drug poisoning, as a side effect of certain drugs, particularly opioids and anticholinergics $[6,8,16]$, or other disorders [8]. Sweating allows the body to regulate its temperature. Sweating is controlled from a center in the preoptic and anterior regions of the brain's hypothalamus, where thermosensitive neurons are located and may be influenced by several factors, humoral ones and drugs for example [2]. Pruritus has been often caused by infections, many medical and dermatologic disorders, allergy, and some medications [9, 19]. Hiccup is caused by nerve damage or irritation, from pre-phrenic nucleus irritation of medulla, or other neurologic disorders [4, 10, 15, 17, 19]. Tenesmus is generally associated with several inflammatory diseases of the bowel and urogenital system or pelvic floor dysfunction [1].

These symptoms are not commonly reported in the most popular and validated symptom assessment tools [3], and may be considered as neglected symptoms. This is the first attempt to assess the prevalence of these orphan symptoms which have never been reported in advanced cancer patients followed at home. These symptoms are really distressing for patients and their families, and the symptom burden has never been assessed.

As expected in this survey, a low prevalence of these symptoms was observed at admission of a home care program. The prevalence of myoclonus was $0.27 \%$, and, consequently, no specific factor could be found to be associated with that. The only patient presenting myoclonus was receiving high doses of oxycodone ( $540 \mathrm{mg}$ /day), haloperidol $5 \mathrm{mg} /$ day, and prednisone $50 \mathrm{mg} /$ day. It is likely that such a symptom may occur in the terminal stage of disease or in conjunction with high doses of opioids, but the number of patients recruited was unexpectedly too small to evidence positive findings. Sweating was more frequent, with $5.24 \%$ of patients presenting this symptom at admission. While the association with fever was expected in some way, a cluster of symptoms, such as hiccup and vesical tenesmus, was unexplainable and may be due to other factors not taken into account or a bias of the sample. Of interest, the presence of sweating should contraindicate the use of transdermal fentanyl which was found to be associated with sweating, due to its unpredictable absorption in such circumstances. And $3.8 \%$ of patients presented hiccup which was more frequently observed in males. This observation has never been reported and provides important data in gender differences in the presentation of hiccup. Pruritus was reported by $3.6 \%$ of patients admitted to home care, but no predictive factors were found, including skin lesions or drugs. Vesical tenesmus was associated with fever and sweating. This relationship deserves further analysis in subsequent larger studies. Unfortunately, comparative findings are unavailable, as no prospective survey has been reporting the prevalence of these symptoms.

The findings of this preliminary investigation show that a minority of patients present the orphan symptoms examined. While these symptoms have a low prevalence in advanced cancer patients admitted to home care, the distress for patients may be high and deserves further analyses to establish the burden, in comparison with other concomitant symptoms, limiting the quality of life. Given the low prevalence of these symptoms, large studies are needed to find possible associated factors.

According to these observations, this study has some limitations, inherent to home care studies and the exploratory nature of the study. A very simple dataset was chosen to facilitate data collection which was partially missed, to provide such preliminary information. Factors analyzed were based on a previous consensus prevalently based on repeatability and simplicity of data to be collected at home. It is likely that the impact of these factors would be better assessed in an even larger sample of patients. Thus, the findings of this survey should be implemented in subsequent large studies. A lack of 
severity rating and influence on quality of life should be eventually assessed. The cross-sectional analysis at time of admission provides only point prevalence, and dynamics of these symptoms should be explored in observational long-term studies. Also, no specific treatment protocol was decided, given the paucity of evidence regarding the treatment of these symptoms in the advanced stage of disease. Thus, no data on the efficacy of consequent treatment was recorded. Data should be repeated a few days before dying to reflect the switch of orphan symptom prevalence close to death [12].

In order to have a terminal cancer patient spending fulfilling remaining of his life, the role of home care is important. While difficult to perform, epidemiological and clinical research at home may provide interesting data about the pattern of orphan symptoms which are uncommonly reported in symptom assessment tool, but may have an important burden for patients and are worthwhile of consideration for a careful monitoring and consequently an appropriate treatment. The preliminary findings of this study should prompt large studies to gather further information on these symptoms.

Acknowledgments We are indebted with the staff of home care programs in Turin, L'Aquila and Genua.

Conflict of interest No conflict of interest to declare.

\section{References}

1. Ang SK (2009) Tenesmus, strangury, and malodor. In Palliative Medicine, Walsh D ed. Saunders, 947-950

2. Bobb B, Lyckholm L, Coyne P (2013) Fever and sweats. In Principles and practice of Palliative Care and Supportive Oncology. Berger AM, Shuster JL, Von Roenn JH eds, Lippincott Williams \& Wilkins, 890-93

3. Bruera E, Kuehn N, Miller MJ, Selmser P, Macmillan K (1991) The Edmonton Symptom Assessment System (ESAS): a simple method for the assessment of palliative care patients. J Palliat Care 7:6-9
4. Calsina-Berna A, García-Gómez G, González-Barboteo J, PortaSales J (2012) Treatment of chronic hiccups in cancer patients: a systematic review. J Palliat Med 15:1142-1150

5. Ewing G, Brundle C, Payne S, Grande G; National Association for Hospice at Home (2012) The Carer Support Needs Assessment Tool (CSNAT) for Use in Palliative and End-of-life Care at Home: A Validation Study. J Pain Symptom Manage

6. Gilbert JE, Howell D, King S, Sawka C, Hughes E, Angus H, Dudgeon D (2012) Quality improvement in cancer symptom assessment and control: the Provincial Palliative Care Integration Project (PPCIP). J Pain Symptom Manage 43:663-678

7. Ito S, Liao S (2008) Myoclonus associated with high-dose parenteral methadone. J Palliat Med 11:838-841

8. Khoshknabi DS (2013) Muscle spasms. In Principles and practice of Palliative Care and Supportive Oncology. Berger AM, Shuster JL, Von Roenn JH eds, Lippincott Williams \& Wilkins, 916-21

9. McCann S, Yaksh TL, von Gunten CF (2010) Correlation between myoclonus and the 3-glucuronide metabolites in patients treated with morphine or hydromorphone: a pilot study. J Opioid Manag 6:87-94

10. Marinella MA (2009) Diagnosis and management of hiccups in the patient with advanced cancer. J Support Oncol 7:122-127

11. Mercadante S (1998) Pathophysiology and treatment of opioidrelated myoclonus in cancer patients. Pain 74:5-9

12. Mercadante S, Valle A, Porzio G, Costanzo BV, Fusco F, Aielli F, Adile C, Fara B, Casuccio A, Home Care-Italy (HOCAI) Group (2011) How do cancer patients receiving palliative care at home die? A descriptive study. J Pain Symptom Manage 42:702-709

13. Nekolaichuk C, Watanabe S, Beaumont C (2008) The Edmonton symptom assessment system: a 15-year retrospective review of validation studies (1991-2006). Palliat Med 22:111-122

14. Richardson LA, Jones GW (2009) A review of the reliability and validity of the Edmonton Symptom Assessment System. Curr Oncol $16: 55$

15. Sarhill N, Mahmoud F (2013) Hiccups and other gastrointestinal symproms. In Palliative care and supportive oncology. WoltesrKluver, Lippincott Williams \& Wilkins, Berger AM, Schuster JL, Von Roenn JH eds. 223-237

16. Seccareccia D, Gebara N (2011) Pruritus in palliative care. Can Fam Physic 57:1010-1014

17. Smith HS (2013) Hiccups. In Principles and practice of Palliative Care and Supportive Oncology. Berger AM, Shuster JL, Von Roenn JH eds, Lippincott Williams \& Wilkins, 895-8.

18. Yelverton C, McGevna (2013) Pruritus. In Principles and practice of Palliative Care and Supportive Oncology. Berger AM, Shuster JL, Von Roenn JH eds, Lippincott Williams \& Wilkins, 309-19

19. Woelk CJ (2011) Managing hiccups. Can Fam Phys 57:672-675 